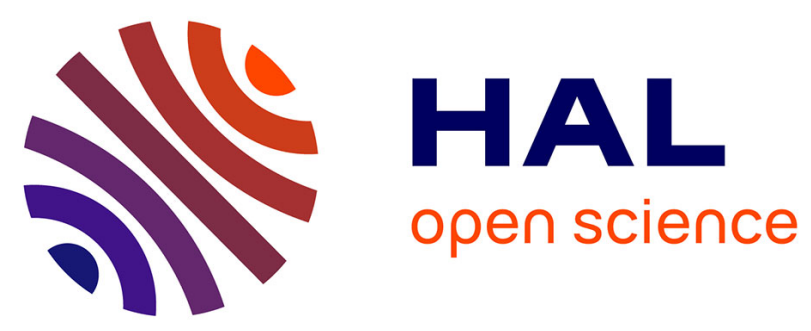

\title{
Multistep Drug Intercalation: Molecular Dynamics and Free Energy Studies of Daunomycin binding to DNA
}

\author{
Matthieu Wilhelm, Arnab Mukherjee, Benjamin Bouvier, Krystyna \\ Zakrzewska, James T. Hynes, Richard Lavery
}

\section{- To cite this version:}

Matthieu Wilhelm, Arnab Mukherjee, Benjamin Bouvier, Krystyna Zakrzewska, James T. Hynes, et al.. Multistep Drug Intercalation: Molecular Dynamics and Free Energy Studies of Daunomycin binding to DNA. Journal of the American Chemical Society, 2012, 134 (20), pp.8588-8596. 10.1021/ja301649k . hal-02395291

\section{HAL Id: hal-02395291 \\ https://hal.science/hal-02395291}

Submitted on 9 Dec 2019

HAL is a multi-disciplinary open access archive for the deposit and dissemination of scientific research documents, whether they are published or not. The documents may come from teaching and research institutions in France or abroad, or from public or private research centers.
L'archive ouverte pluridisciplinaire HAL, est destinée au dépôt et à la diffusion de documents scientifiques de niveau recherche, publiés ou non, émanant des établissements d'enseignement et de recherche français ou étrangers, des laboratoires publics ou privés. 


\title{
Multistep drug intercalation: molecular dynamics and free energy studies of daunomycin binding to DNA
}

\author{
Matthieu Wilhelm', Arnab Mukherjee², Benjamin Bouvier', Krystyna Zakrzewska', \\ James T. Hynes ${ }^{3,4}$, Richard Lavery' \\ 'Bioinformatics: Structures and Interactions, Bases Moléculaires et Structurales des Systèmes \\ Infectieux, Univ. Lyon I / CNRS UMR 5086, IBCP, 7 Passage du Vercors, Lyon 69367, France \\ ${ }^{2}$ Chemistry Department, Indian Institute of Science Education and Research, Pune, 411021, India \\ ${ }^{3}$ Department of Chemistry and Biochemistry, University of Colorado, Boulder, CO 80309-0215, USA \\ ‘Chemistry Department, Ecole Normale Supérieure, CNRS UMR 8640, 24 rue Lhomond, 75005 Paris, \\ France
}

KEYWORDS intercalation, drub binding, daunomycin, nucleic acid, molecular dynamics, metadynamics, umbrella sampling, free energy, transition states

\begin{abstract}
Atomic-scale molecular dynamics and free energy calculations in explicit aqueous solvent are used to study the complex mechanism by which a molecule can intercalate between successive base pairs of the DNA double helix. We have analyzed the intercalation pathway for the anticancer drug daunomycin using two different methods: metadynamics and umbrella sampling. The resulting free energy pathways are found to be consistent with one another and point to a three-step process. Duanomycin initially binds in the minor groove of DNA. An activated step then leads to rotation of the drug, coupled with DNA deformation that opens a wedge between the base pairs and forms an unexpected metastable intermediate. Crossing a small energy barrier causes further rotation and full intercalation of the drug and reestablishes stacking within the double helix.
\end{abstract}




\section{INTRODUCTION}

An important class of pharmacologically active molecules function by intercalation between the base pairs of DNA, subsequently interfering with DNA function or replication, often by blocking the action of topoisomerases. Intercalators are characterized by planar, conjugated, fused ring systems, whose dimensions resemble DNA base pairs. Insertion between consecutive base pairs shields the hydrophobic rings of the intercalator from water, while maintaining favorable $\pi$-stacking within the double helix. ${ }^{12}$ Despite the medical applications of intercalators, and many years of study, the detailed mechanism of the intercalation process has not yet been determined. ${ }^{34}$ As structural studies have shown, intercalation must clearly lengthen DNA, doubling the inter-BP rise at the intercalation site (from approximately $3.4 \AA$ to $6.8 \AA$ ), and generally reducing twist, but the pathway (or pathways) leading to this state from the free intercalator in solutions are unknown. Kinetic studies point to a complex multi-step process..$^{5-13}$ Notably, for the final intercalation step, current evidence suggests that the intercalator is involved in creating the intercalation site, rather than passively waiting for such a site to occur spontaneously, ${ }^{14.16}$ despite the fact that significant fluctuations in DNA rise are known to occur at room temperature. ${ }^{17}$

Here, we use atomic-resolution molecular dynamics in aqueous solution, coupled with two different free energy evaluation procedures to study the details of the intercalation process for daunomycin (also know as daunorubicin), ${ }^{18.19}$ an anticancer, anthracyclin drug ${ }^{20}$ that binds to DNA and blocks its replication. The structure of DNA-daunomycin complexes have been resolved by crystallography ${ }^{21.24}$ and by NMR spectroscopy ${ }^{25,26}$ and show the characteristic features of most intercalating drugs. Kinetic studies of daunomycin by Chaires et al. ${ }^{10}$ suggested a three-step model for intercalation, involving an initial "outside" binding to DNA and a final "reshuffling" of the drug within the intercalation site. Other studies by Rizzo et al. suggested a fivestep process..$^{13}$ Using detailed simulations, we hope to be able to clarify the structural and energetic features of the intercalation pathway and to identify any intermediate states, providing information that cannot easily be obtained with current experimental techniques.

This work builds on our previous studies of daunomycin binding to DNA that revealed a stable, minor groove-bound state. ${ }^{27}$ This state did not require significant 
DNA deformation and could explain the "outside" bound step postulated by Chaires and coworkers. ${ }^{10}$ However, while our former study identified some key motions of the DNA-daunomycin-water system involved in the transition from the groove-bound to the intercalated state, and estimated the free energy barrier, the methodology employed could not provide a detailed description of the transition. The present study fills this gap.

Since intercalation is a conformationally complex process, involving significant DNA deformation coupled with a major repositioning of the drug molecule, we have derived optimal free energy pathways with two distinct methods. The first uses non-equilibrium, metadynamics simulations ${ }^{28}$ in a specifically-designed collective variable space. Metadynamics enables a system to sample large volumes of conformational space in a reasonable computational time by regularly adding local destabilizing potentials to the natural energy hypersurface. It has already been successfully used to study a variety of binding and unbinding processes.$^{2.931}$ The second method uses equilibrium umbrella sampling with the aid of a biasing potential to drive the system between the desired states in regular steps. In order to study the separation of two molecules using this technique, we have recently developed a new restraint that avoids having to specify a separation pathway. This approach has already been successfully used to study the mechanism of protein-DNA unbinding. ${ }^{32,33}$ The results of these two independent approaches are found to be consistent and reveal a passage from the groove to the intercalation site involving a metastable intermediate and two transition states.

\section{METHODOLOGY}

Simulation setup Following the procedure set out in our earlier study, ${ }^{27}$ the initial conformation of daunomycin, and of its DNA intercalation site, were drawn from crystallographic results, ${ }_{, 23}^{23}$ using the PDB structure 1D11. Since this structure contains two daunomycin molecules, we extracted a single $\mathrm{CpG}$ intercalation site, including one daunomycin, contained within a three nucleotide pair 5'-ACG-3' segment. The 5'AT base pair was retained because the charged sugar moiety of daunomycin (see Figure 1) lies in the minor groove, close to this base pair. The ACG segment was then 
extended using JUMNA, ${ }^{34}$ by adding canonical B-DNA arms on either side to form a double-stranded dodecamer with the sequence d(GCGCACGTGCGC), where the underline indicates the position of the central C6pG7 intercalation site. Note that a CpG step (that is a CG base pair followed by a GC base pair in the 5'-3' sense) has been shown to be the strongest binding site for daunomycin. ${ }^{35.37}$ Note also that the dodecamer has a palindromic base sequence. This implies that the orientations of the drug created by $180^{\circ}$ rotations around the $\mathrm{C} 5-\mathrm{C} 12$ axis of the anthraquinone segment (see Figure 1), either in the intercalation site, or centrally-positioned in a DNA groove, will be symmetrically equivalent.

Simulation protocol DNA was simulated using the AMBER parm99/bsc0 force field $^{3339}$ that corrects earlier problems connected with unusual backbone states and leads to conformational distributions in good agreement with experiment. ,940 $^{34}$ Daunomycin was represented using the general AMBER force field (GAFF) ${ }^{41}$ Its atomic charges were calculated with the Merz-Kollman electrostatic potential potential-fitting procedure ${ }^{42}$ in the Gaussian quantum chemistry package, ${ }^{43}$ using a Hartee-Fock wavefunction obtained with a $6-31 \mathrm{G}^{*}$ basis set for compatibility with the partial charges used for DNA in the AMBER force field.

The initial conformation of the daunomycin-DNA complex was simulated in a cubic box with periodic boundary conditions. The solute was surrounded by roughly 9000 TIP3P water molecules,,$^{44}$ leaving at least $10 \AA$ space between the solute and the faces of the box. The solute charges were compensated in two possible ways, either by adding a minimal number of ions to neutralize the system $\left(22 \mathrm{Na}^{+}\right.$and $\left.1 \mathrm{Cl}\right)$, or by adding sufficient additional salt $(\mathrm{NaCl})$ to reach a concentration of $0.15 \mathrm{M}$, close to physiological conditions. In both cases, ion parameters from Dang et al. were used.4 The system was equilibrated using energy minimization followed by careful heating to $300 \mathrm{~K}$ with harmonic restraints on the solute atom positions. These restraints were relaxed in a series of energy minimizations, followed by short molecular dynamics simulations. The system was then equilibrated for $5 \mathrm{~ns}$, with no positional restraints, and using an isothermal-isobaric ensemble. Subsequent production simulations were carried out using either the GROMACS ${ }^{46}$ or NAMD ${ }^{47}$ program suites. All simulations used a 2 fs time step. For the metadynamics simulations, iterative restraints were 
applied to all bond lengths, while for the umbrella-sampling dynamics simulations only $\mathrm{X}-\mathrm{H}$ bond lengths (where $\mathrm{X}$ is any heavy atom) were restrained.

Metadynamics free energy pathways The first pathway generation approach uses metadynamics, which is a non-equilibrium sampling method, carried out using chosen collective variables. In order to generate a pathway between the intercalated and groove-bound states of daunomycin, we use three collective variables: $\mathrm{X}$ which describes the distance of the drug from DNA, Y which describes its movement in the direction of the DNA helical axis and $\theta$ which describes the orientation of the planar anthraquinone moiety. $X$ and $\theta$ are the same variables used in our earlier study. ${ }^{27}$ The geometrical definition of these variables is given in the Supplementary Information. We also follow the procedure of this publication in examining the intercalation process in the reverse sense. In order to make the drug move away from the intercalation site as the simulation progresses, destabilizing Gaussian potentials are added to the region of collective variable space currently being sampled, thus forcing the system to explore higher energy regions. When convergence is reached, the Gaussian potentials allow the underlying free energy surface to be reconstructed. Metadynamics was carried out with GROMACS using the GROMETA implementation ${ }^{48}$ Gaussian potentials were added every 0.2 ps with a height of 0.024 $\mathrm{kcal} \mathrm{mol}^{-1}$ and widths of $0.1 \AA$ in the $\mathrm{X}$ and $\mathrm{Y}$ coordinates and of 0.2 radians in the $\theta$ coordinate. Several independent simulations were carried out in 1D (using the $X$ coordinate), in 2D (using the $\mathrm{X}$ and $\theta$ coordinates) and in $3 \mathrm{D}$ (using the $\mathrm{X}, \theta$ and $\mathrm{Y}$ coordinates). We present here only the 3D simulations that required of the order of $100 \mathrm{~ns}$ of sampling.

Umbrella sampling free energy pathways The second pathway generation approach uses umbrella sampling. This is an equilibrium technique that constrains the system at a series of intermediate steps along a chosen coordinate using a biasing potential. Providing the sampling at successive steps overlaps, the impact of the biasing potential can be removed using the weighted-histogram analysis method, WHAM ${ }^{49}$ In order to separate daunomycin from DNA without imposing a specific spatial coordinate, we replaced the specific coordinates used in our earlier work ${ }^{27}$ with a more general approach developed for studying macromolecular dissociation processes..$^{32}$ This involves a restraint on the minimal approach distance between any pair of non- 
hydrogen atoms at the interface between two molecules. Any atom pair falling below the chosen distance limit leads to a quadratic penalty energy and consequently to atomic forces pushing the pair apart to the desired minimal distance. To avoid the two molecules drifting apart once they no longer interact strongly, we also impose a similar quadratic penalty on the closest atom pair at the interface, if this pair lies beyond the desired distance. By increasing the restraint distance in small steps $(0.15$ $\AA$ ) and adequately sampling the corresponding conformational fluctuations of the system (1-2 ns sampling before changing the restraint distance, followed by 2-4 ns of further simulation) we can build up an accurate view of the free energy pathway.

We also used umbrella sampling to analyze the internal flexibility of daunomycin, and, in particular, of its flexible A-ring. This was sampled using the C7C8-C9-C10 dihedral angle (hereafter termed $\tau$ ) to scan the possible conformations of this ring. The dihedral was changed in $5^{\circ}$ steps and restrained with a force constant of $0.15 \mathrm{kcal} \mathrm{mol}^{-1} \mathrm{deg}^{-1}$. We performed $1 \mathrm{~ns}$ of sampling before each change. Each point was then sampled for a further $3 \mathrm{~ns}$. The 30 sampling windows along each pathway required $120 \mathrm{~ns}$ of simulation. The corresponding free energy curves were again obtained using the WHAM procedure..$^{9}$

Conformational analysis A variety of tools were used for conformational analysis. The overall characteristics of molecular dynamics trajectories were analyzed using structures ("snapshots") extracted at regular 1 ps intervals. These structures were superposed..$^{50}$ and compared using root mean square difference (RMSD) calculations restricted to non-hydrogen atoms. Representative conformations at a given points along the free energy pathways were chosen as the structure closest to the average structure of the snapshots sampled at this point, again using RMSD calculations. DNA structure was analyzed using the Curves+ program $^{s 1}$ which was extended for the present study by including the possibility of locating a DNA-bound ligand. This enables a reference frame on the ligand to be located with respect to a reference frame on the DNA helical axis. Here, the position of daunomycin with respect to the center of the DNA helical axis is simplified to two parameters, the overall translation (LTra) and the overall rotation (L-Rot) between the two frames (see Supplementary Information for details). 
All molecular representations in this work were prepared using VMD, $2 \mathrm{D}$ and 3 D graphics were prepared with Gnuplot, Mathematica ${ }^{\circledR}$ and MatLab®.

\section{RESULTS}

\section{i) Metadynamics intercalation pathway}

Following a $110 \mathrm{~ns}$ metadynamics trajectory in the three collective variables described above $(\mathrm{X}, \theta, \mathrm{Y})$, and using minimal salt conditions, we extracted a $2 \mathrm{D}$ free energy surface by averaging over the values along the Y coordinate. The results in Figure 2 show several local free energy minima that we have labeled IC, IM, MG1, MG2 and MG3. A dotted line indicates the lowest free energy path between these minima and indicates the associated transition states, labeled TS1-TS4. IC corresponds to the intercalated state of daunomycin, while MG1, MG2, and MG3 all have the drug lying within the minor groove. MG1 is the deepest of these minima, and we not discuss the other groove-bound minima further since drug movement within the minor groove is still under investigation and is not our focus here.

The most striking feature of Figure 2 is a well-defined free energy basin corresponding to an intermediate state IM, lying between the highest transition states. This minimum is reached after daunomycin has moved roughly $4.5 \AA$ along $\mathrm{X}$, away from the intercalated state IC, and has rotated by roughly $40^{\circ}$ in the $\theta$ coordinate. As shown in Table 1, IM lies $7.3 \mathrm{kcal} \mathrm{mol}^{-1}$ above IC and $6.9 \mathrm{kcal} \mathrm{mol}^{-1}$ above the best minor groove-bound state MG1. The intermediate state is flanked by two transition states, TS1, $2.6 \mathrm{kcal} \mathrm{mol}^{-1}$ above IM, leading to the intercalation site, and TS2, 2.4 $\mathrm{kcal} \mathrm{mol}^{-1}$ above IM, leading to the minor groove site.

Figure 3 (top) illustrates representative structures along the metadynamics intercalated to minor groove-bound pathway. These were obtained as the average structures of clusters ${ }^{53}$ lying within $2 \AA$ RMSD of each significant point (transition state of free energy minimum) along the pathway. Looking again at Figure 2, we see that the drug initially moves mainly in the X-direction, since its anthraquinone moiety is still held between the DNA base pairs. Once this interaction weakens, the drug begins to rotate (decreasing $\theta$ ) and DNA bends away from daunomycin, creating a 
large positive roll at the $\mathrm{CpG}$ step in order to regain some favorable stacking between these base pairs (see the top central image of Figure 3). This is the situation at the intermediate IM state. As the drug moves slightly further away and continues to rotate, DNA can relax by recovering full $\mathrm{CpG}$ stacking, while at $\theta \approx 80^{\circ}$, the drug finally locates its optimal position within the minor groove. The main conformational parameters of the states along the metadynamics pathway are given in Table 2.A.

\section{ii) Umbrella sampling of daunomycin conformations}

Since the metadynamics pathway showed that the conformation of daunomycin changes as the drug moves from the intercalation site to the groove (see Table 2.A), we began the umbrella sampling studies by analyzing the internal flexibility of daunomycin, and notably the properties of its non-planar A-ring. It has been shown experimentally that this ring favors two pucker states that can be characterized by the torsion angle $\tau$ (C7-C8-C9-C10). $\tau \approx-60^{\circ}$ places the C13 acetyl group in an axial position, while $\tau \approx+50^{\circ}$ places it in an equatorial position..$^{5458}$. The most recent experiments find that the equatorial state is favored in solution, although the axial state is observed in the presence of divalent ions..$^{90}$

We have studied the repuckering of the A-ring in three different environments: free in aqueous solution, in the intercalated state (IC) and in the minor groove-bound state (MG1). The corresponding free energy curves were obtained by umbrella sampling of the torsion angle $\tau$, initially used minimal salt conditions as for the metadynamics studies described in section (i). As expected, they all show an axial minimum close to $\tau=-60^{\circ}$ and an equatorial minimum close to $\tau=+50^{\circ}$. When daunomycin is isolated (Figure 4.A), these two states are almost equally stable and are separated by a barrier of $\leq 3 \mathrm{kcal} \mathrm{mol}^{-1}$. In contrast, the minor groove-bound state (Figure 4.B), shows a clear preference for the axial conformation (1.5 kcal mol-1), coupled with a larger barrier separating the two states $\left(\geq 4 \mathrm{kcal} \mathrm{mol}^{-1}\right)$. Lastly, in the intercalated site, the DNA environment strongly favors an equatorial conformation (Figure 4.C), in agreement with crystallographic observations, ${ }^{26}$ and any values of $\tau$ $<+20^{\circ}$ are disfavored by at least $6 \mathrm{kcal} \mathrm{mol}^{-{ }^{-}}$.

During this study, we noticed that changing the A-ring puckering in the minor groove-bound state led to a strong coupling with the minor groove width, which 
increased by roughly $3 \AA$ as daunomycin transited to the wider axial state (Figure S2.A). This led us to wonder whether minimal salt conditions were exaggerating the role of interactions between DNA and the charged daunosamine sugar of the drug. We consequently repeated the free energy calculations using a $0.15 \mathrm{M} \mathrm{NaCl}$ concentration, close to physiological conditions. This indeed led to a decoupling of the minor groove width (see Figures S2.B) and significant change in the free energy profile (see Figure 4.B, blue curve), which now favored the equatorial conformation of the A-ring. The free energy profiles for free or intercalated daunomycin showed less change with a physiological salt concentration and did not affect the preferred Aring pucker, however, given the impact on the minor groove width, we decided to use physiological salt conditions for the umbrella-sampling studies of daunomycin-DNA intercalation pathway.

\section{iii) Umbrella sampling intercalation pathway}

Umbrella sampling was used to construct a single pathway between the intercalated state and the groove-bound state from two separate pathways, one removing daunomycin from its intercalation site (the IC-pathway) and the other removing daunomycin from its minor groove-bound site (the MG1-pathway). These two pathways were found to intersect and can thus be used to build a single IC - MG1 free energy profile.

The IC-pathway removed daunomycin from its intercalation site using a separation restraint acting on the rigid part of the A-ring (atoms C7-C6a-C10a-C10) and the atoms of the two base pairs forming the intercalation site. This choice ensured movement towards the minor groove side of the intercalation site, while leaving the ligand free to reorient once was no longer stacked with the flanking bases. This restraint had a value of $3.2 \AA$ for the IC state. Umbrella sampling was carried out over the range $3.1-12.5 \AA$ in $0.15 \AA$ steps with $4-8 \mathrm{~ns}$ of sampling at each point (more sampling was used in regions where large conformational changes occurred either in DNA or in the orientation of the drug).

The resulting free energy profile (Figure 5.A) shows a sharp, linear free energy rise as the anthraquinone ring system is removed from the DNA base stack. At $5.8 \AA$, daunomycin begins to rotate, reflected by a local energy maximum $9 \mathrm{kcal} \mathrm{mol}^{-1}$ 
above the free energy of the intercalated state, followed by a metastable intermediate state at $6.8 \AA$, with a free energy of $7.7 \mathrm{kcal} \mathrm{mol}^{-1}$. This intermediate lies within a quadratic energy well that ends at roughly $7.4 \AA$. Further separation of the drug leads to a slower, more or less linear energy rise. The final free energy is $13 \mathrm{kcal} \mathrm{mol}^{-1}$ above the IC state, but it is important to note that complete sampling is very difficult once the ligand moves away from DNA and is free to tumble.

A 2D RMSD plot of representative snapshots drawn from this free energy profile (Figure 6.A) confirms the existence of five conformational clusters along the pathway: (a) intercalated or partially intercalated; (b) first transition state; (c) metastable intermediate: (d) second transition state; (e) separation. Note that the intermediate state flanked by two transition states was not observed in our earlier less detailed study of the reaction pathway. ${ }^{27}$

A second umbrella-sampling pathway, the MG1-pathway, was used to remove daunomycin from the minor groove-bound state that we constructed during our earlier studies. ${ }^{27}$ This involved a separation restraint acting on all heavy atoms of the ligand and on all base atoms of DNA. The restraint had a value of $2.8 \AA$ for the MG1 state. Umbrella sampling was carried out over the range $2.5-7.1 \AA$ in $0.15 \AA$ steps with 6 ns of MD sampling at each step. Figure 5.B shows an approximately quadratic free energy profile around the minimum, followed by a more linear free energy rise starting from a separation of $3.5 \AA$, when attractive interactions between daunomycin and the sides of the minor groove are lost. The changes which occur at this point are reflected in the 2D RMSD plot shown in Figure 6.B.

As shown by the 2D RMSD plot in Figure 6.C, it is in fact possible to link the IC- and MG1-pathways into a single IC - MG1pathway. Indeed, we find a very close correspondence between the structures at a separation of $7.25 \AA$ on the IC pathway and those at a separation of $4.1 \AA$ on the MG1 pathway. We recall that these two distances differ significantly because they refer to different choices of interface atoms for the restraint.

Joining the two free energy pathways allows us to place the minor groove minimum at a free energy $2.7 \mathrm{kcal} \mathrm{mol}^{-1}$ relative to the intercalated state (see Figures 5.C and 5.D). Representative structures along the IC - MG1 pathway, including the 
intermediate free energy minimum IM and the two flanking transition states, TS1 and TS2, are illustrated in Figure 3 (bottom line) and their main conformational parameters are given in Table 2.B.

\section{iv) Comparison of metadynamics and umbrella results}

A first comparison of the metadynamics and umbrella sampling IC - MG1 pathways can be made using the free energy values in Table 1 and the conformational parameters in Table 2. The free energies of the significant points along both pathways are very similar to one another. The differences are almost certainly below the accuracy of the calculations, with the possible exception of the minor-groove bound state MG1. The umbrella-sampling pathway finds this state to be $2.7 \mathrm{kcal} \mathrm{mol}^{-1}$ less stable than the intercalated state (compared to only $0.4 \mathrm{kcal}^{\mathrm{mol}{ }^{-1}}$ using metadynamics). We attribute this to the use of a physiological salt concentration for the umbrella-sampling simulations, since these conditions diminish the importance of the ionic interactions between the daunosamine sugar and DNA backbone. This result also seems more in line with the fact that the minor groove-bound state is only an intermediate preceding intercalation. The physiological salt concentration may also be responsible for slightly lowering the energies of the transition states on either side of the IM intermediate.

The conformational parameters for the states along the two pathways are given in Table 2, and, as for the free energies discussed above, they follow similar trends. The IC and MG1 states are similar (except for a narrower minor groove for the umbrella-sampling MG1 state). Some differences occur for IM and the two flanking transition states, although these are not very evident from the conformational snapshots shown in Figure 3, apart from differences in the daunomcycin orientation. Different salt conditions may again explain these differences since, as we have seen, using added salt changes affects the puckering of the A-ring. This impact is visible for the ring conformations listed in Table 2 and may consequently modify the optimal positioning of daunomycin and indirectly influence DNA deformation.

For a more global comparison of the metadynamics and umbrella-sampling results we have performed two further analyses. The first uses 2D RMSD plots comparing representative conformations drawn from the two pathways. As Figure 7 
shows, the agreement is good for the significant points along the pathway, both for the DNA alone (RMSD $<2 \AA$ ) and for the DNA-daunomycin complex (RMSD $<2.5$ $\AA$ ), with the exception of the intermediate IM state where the inclination of the drug changes between the two pathways. In fact, some of these differences are probably linked to the choice of representative conformations, given that determining the exact position of the significant points along the pathway is limited by the accuracy of the free energy curves.

The second, and more continuous, view of the pathways is given in the $3 \mathrm{D}$ plot in Figure 8 that combines information on DNA structure (namely, the rise and roll at the $\mathrm{C} 6 \mathrm{pG} 7$ intercalation step), information on the ligand position (the distance from the center of the DNA helical axis) and free energy (colored blue to green as free energy increases). This scatter plot contains more than 15,000 snapshots from the umbrella-sampling IC - MG1 path which clearly visualize the clusters of points associated with the intercalated state IC (bottom), the intermediate state IM (center right) and the groove-bound state MG1 (top). The lines between the significant points along the pathway show that the metadynamics path (open circles) and the umbrellasampling path (filled circles) are very similar in the chosen variables. The differences in the IM state due to daunomycin orientation mentioned above do not show up here as only DNA-drug distance is plotted. These results also show that the transition states, TS1 and TS2, occur closer to IM in the metadynamics pathway than in the umbrella-sampling pathway. However, conformational changes occur very rapidly during these transitions as witnessed by the sparsity of sampling in between the IC, IM and MG1 regions.

\section{CONCLUSIONS}

By using two different free energy sampling methods we have obtained a consensus view of how the drug daunomycin intercalates within the base stack of a DNA double helix. The optimal pathway involves initial binding to the minor groove with minimal DNA deformation (with a binding energy of roughly $-10 \mathrm{kcal} \mathrm{mol}^{-1}$ using the umbrella-sampling simulations with a physiological salt concentration). An activated process (requiring roughly $6.5 \mathrm{kcal} \mathrm{mol}^{-1}$ under the same conditions) causes the drug to rotate and to partially insert its planar anthraquinone moiety into a wedge formed between two successive base pairs. This intermediate state lies in a shallow 
free energy well (with a depth of roughly 1.5-2 $\mathrm{kcal} \mathrm{mol}^{-1}$ ). Crossing a small energy barrier is therefore sufficient to achieve full intercalation.

If we consider the dissociation reaction, then the simulations indicate a $9 \mathrm{kcal}$ mol $^{-1}$ barrier to reach the intermediate state, followed again by a small barrier of 1.5-2 $\mathrm{kcal} \mathrm{mol}^{-1}$ to reach the minor groove site, and roughly $10 \mathrm{kcal} \mathrm{mol}^{-1}$ to escape into solution. This result is partially compatible with the 3-state model of Chaires..$^{10,60}$ If we associate the first "outside-bound" state with our minor groove binding and the second state with the intercalated state, then the activation energies (estimated from the temperature dependence of the corresponding rate constants) for escaping from the intercalation site of $\left(14.3 \mathrm{kcal} \mathrm{mol}^{-1}\right)$ and then from the minor groove site $(10.7$ $\mathrm{kcal} \mathrm{mol}^{-1}$ ) roughly fit with our data. The second barrier to escape the intermediate state, which does not appear in the 3-state model, ${ }^{10}$ might explain the larger single activation energy seen for this step. However, we see no process that could explain the third step involving "a conformational rearrangement of the intercalated drug molecule or the DNA molecule" (with a surprisingly high activation energy for escaping from this third state of $\left.16.2 \mathrm{kcal} \mathrm{mol}^{-1}\right)^{10}$ other than a repositioning the drug between another base pair. ${ }^{27}$ Further studies will be necessary to characterize drug movements along the minor groove and eventual passages from one intercalation site to another.

\section{ACKNOWLEDGEMENTS}

The authors acknowledge funding from the ANR Blanc project ALADDIN and CINES for the allocation of supercomputer resources. BB, KZ and RL thank the CNRS for their support. This work was supported in part by NSF grants CHE0750477 and CHE-1112564 (JTH). AM and JTH acknowledge TeraGrid for supercomputing facilities. AM also thanks computational facility of IISER, Pune.

\section{REFERENCES}

(1) Lerman, L. S. Journal of molecular biology. 1961, 3, 18-IN14.

(2) Strekowski, L. and Wilson, B. Mutat Res. 2007, 623, 3-13.

(3) Müller, W. and Crothers, D. M. Journal of molecular biology. 1968, 35, 251. 
(4) Chaires, J. B.; Dattagupta, N.; and Crothers, D. M. Biochemistry. 1982, 21, 3933 3940.

(5) Li, H. J. and Crothers, D. M. J Mol Biol. 1969, 39, 461-77.

(6) Chaires, J. B. Biochemistry. 1983, 22, 4204-4211.

(7) Graves, D. E. and Krugh, T. R. Biochemistry. 1983, 22, 3941-3947.

(8) Forster, W. and Stutter, E. International Journal of Biological Macromolecules. 1984, 6, 114-124.

(9) Wilson, W. D.; Krishnamoorthy, C. R.; Wang, Y. H.; and Smith, J. C. Biopolymers. 1985, 24, 1941-1961.

(10) Chaires, J. B.; Dattagupta, N.; and Crothers, D. M. Biochemistry. 1985, 24, 260267.

(11) Krishnamoorthy, C. R.; Yen, S. F.; Smith, J. C.; Lown, J. W.; and Wilson, W. D. Biochemistry. 1986, 25, 5933-5940.

(12) Macgregor, R. B. J.; Clegg, R. M.; and Jovin, T. M. Biochemistry. 1987, 26, 4008-16.

(13) Rizzo, V.; Sacchi, N.; and Menozzi, M. Biochemistry. 1989, 28, 274-82.

(14) Ramstein, J.; Dourlent, M.; and Leng, M. Biochemical and biophysical research communications. 1972, 47, 874-882.

(15) Bresloff, J. L. and Crothers, D. M. Journal of molecular biology. 1975, 95, 103110.

(16) Corin, A. F. and Jovin, T. M. Biochemistry. 1986, 25, 3995-4007.

(17) Leger, J. F.; Robert, J.; Bourdieu, L.; Chatenay, D.; and Marko, J. F. Proc Natl Acad Sci U S A. 1998, 95, 12295-9.

(18) DiMarco, A.; Gaetani, M.; Orezzi, P.; Scarpinato, B. M.; Silvestrini, R.; Soldati, M.; Dasdia, T.; and Valentini, L. Nature. 1964, 201, 706.

(19) Myers Jr, C. E. and Chabner, B. A. Lippincott: Philadelphia, PA. 1990, 256-381. 
(20) Minotti, G.; Menna, P.; Salvatorelli, E.; Cairo, G.; and Gianni, L. Pharmacological reviews. 2004, 56, 185-229.

(21) Tsai, C. C.; Jain, S. C.; and Sobell, H. M. Proceedings of the National Academy of Sciences. 1975, 72, 628.

(22) Quigley, G. J.; Wang, A. H.; Ughetto, G.; Van Der Marel, G.; Van Boom, J. H.; and Rich, A. Proceedings of the National Academy of Sciences. 1980, 77, 7204.

(23) Wang, A. H. J.; Ughetto, G.; Quigley, G. J.; and Rich, A. Biochemistry. 1987, 26, 1152-1163.

(24) Nunn, C. M.; Van Meervelt, L.; Zhang, S.; Moore, M. H.; and Kennard, O. Journal of molecular biology. 1991, 222, 167-177.

(25) Davies, D. B.; Eaton, R. J.; Baranovsky, S. F.; and Veselkov, A. N. Journal of biomolecular structure \& dynamics. 2000, 17, 887.

(26) Barthwal, R.; Sharma, U.; Srivastava, N.; Jain, M.; Awasthi, P.; Kaur, M.; Barthwal, S. K.; and Govil, G. Eur J Med Chem. 2006, 41, 27-39.

(27) Mukherjee, A.; Lavery, R.; Bagchi, B.; and Hynes, J. T. J Am Chem Soc. 2008, $130,9747-55$.

(28) Laio, A. and Parrinello, M. Proc Natl Acad Sci U S A. 2002, 99, 12562-6.

(29) Gervasio, F. L.; Laio, A.; and Parrinello, M. Journal of the American Chemical Society. 2005, 127, 2600-2607.

(30) Pietrucci, F.; Marinelli, F.; Carloni, P.; and Laio, A. Journal of the American Chemical Society. 2009, 131, 11811-11818.

(31) Vargiu, A. V.; Ruggerone, P.; Magistrato, A.; and Carloni, P. Nucleic Acids Res. 2008, 36, 5910-21.

(32) Bouvier, B. and Lavery, R. J Am Chem Soc. 2009, 131, 9864-5.

(33) Bouvier, B.; Zakrzewska, K.; and Lavery, R. Angew Chem Int Ed Engl. 2011, 6516-6518. 
(34) Lavery, R.; Zakrzewska, K.; and Sklenar, H. Computer Physics Communications. 1995, 91, 135-158.

(35) Chen, K. X.; Gresh, N.; and Pullman, B. Journal of biomolecular structure \& dynamics. 1985, 3, 445 .

(36) Chaires, J. B.; Herrera, J. E.; and Waring, M. J. Biochemistry. 1990, 29, 61456153.

(37) Roche, C. J.; Thomson, J. A.; and Crothers, D. M. Biochemistry. 1994, 33, 92635 .

(38) Wang, J. M.; Cieplak, P.; and Kollman, P. A. Journal of Computational Chemistry. 2000, 21, 1049-1074.

(39) Pérez, A.; Marchán, I.; Svozil, D.; Sponer, J.; Cheatham, T. E.; Laughton, C. A.; and Orozco, M. Biophys J. 2007, 92, 3817-29.

(40) Lavery, R.; Zakrzewska, K.; Beveridge, D.; Bishop, T. C.; Case, D. A.; Cheatham, T.; Dixit, S.; Jayaram, B.; Lankas, F.; Laughton, C.; Maddocks, J. H.; Michon, A.; Osman, R.; Orozco, M.; Perez, A.; Singh, T.; Spackova, N.; and Sponer, J. Nucleic Acids Res. 2010, 38, 299-313.

(41) Wang, J.; Wolf, R. M.; Caldwell, J. W.; Kollman, P. A.; and Case, D. A. Journal of computational chemistry. 2004, 25, 1157-1174.

(42) Besler, B. H.; Merz Jr, K. M.; and Kollman, P. A. Journal of Computational Chemistry. 1990, 11, 431-439.

(43) Frisch, M. J.; Trucks, G. W.; Schlegel, H. B.; Scuseria, G. E.; Robb, M. A.; Cheeseman, J. R.; Montgomery Jr, J. A.; Vreven, T.; Kudin, K. N.; and Burant, J. C. Gaussian Inc.: Wallingford, CT. 2003.

(44) Jorgensen, W. L. and Jenson, C. Journal of Computational Chemistry. 1998, 19, $1179-1186$.

(45) Dang, L. X. Journal of the American Chemical Society. 1995, 117, 6954-6960.

(46) Hess, B.; Kutzner, C.; van der Spoel, D.; and Lindahl, E. J. Chem. Theory Comput. 2008, 4, 435-447. 
(47) Bhatele, A.; Kumar, S.; Mei, C.; Phillips, J. C.; Zheng, G.; and Kale, L. V. 2009.

(48) Camilloni, C.; Provasi, D.; Tiana, G.; and Broglia, R. A. Proteins: Structure, Function, and Bioinformatics. 2008, 71, 1647-1654.

(49) Kumar, S.; Bouzida, D.; Swendsen, R. H.; Kollman, P. A.; and Rosenberg, J. M. Journal of Computational Chemistry. 1992, 13, 1011-1021.

(50) McLachlan, A. D. Eur J Biochem. 1979, 100, 181-7.

(51) Lavery, R.; Moakher, M.; Maddocks, J. H.; Petkeviciute, D.; and Zakrzewska, K. Nucleic Acids Res. 2009, 37, 5917-5929.

(52) Humphrey, W.; Dalke, A.; and Schulten, K. J Mol Graph. 1996, 14, 33-8.

(53) Daura, X.; Gademann, K.; Jaun, B.; Seebach, D.; Van Gunsteren, W. F.; and Mark, A. E. Angewandte Chemie International Edition. 1999, 38, 236-240.

(54) Nuss, M. E.; James, T. L.; Apple, M. A.; and Kollman, P. A. Biochimica et Biophysica Acta (BBA)-Nucleic Acids and Protein Synthesis. 1980, 609, 136-147.

(55) Lown, J. W. and Chen, H. H. Canadian Journal of Chemistry. 1981, 59, 32123217.

(56) Mondelli, R.; Ragg, E.; Fronza, G.; and Arnone, A. J. Chem. Soc., Perkin Trans. 2. 1987, 15-26.

(57) Barthwal, R.; Mujeeb, A.; Srivastava, N.; and Sharma, U. Chemico-biological interactions. 1996, 100, 125-139.

(58) Barthwal, R.; Srivastava, N.; Sharma, U.; and Govil, G. Journal of Molecular Structure. 1994, 327, 201-220.

(59) Myung, J. M.; Jhon, M. S.; and Kang, Y. K. Bull. Korean Chem. Soc. 1987, 8.

(60) Chaires, J. B. Biophysical chemistry. 1990, 35, 191-202. 
Table 1. Free energy differences $\left(\mathrm{kcal} \mathrm{mol}^{-1}\right)$ with respect to the intercalated state along the metadynamics and umbrella-sampling paths towards the minor groove bound state.

\begin{tabular}{|l|l|l|l|l|l|}
\hline Pathway & IC & TS1 & IM & TS2 & MG1 \\
\hline Metadynamics & 0 & 9.9 & 7.3 & 9.7 & 0.4 \\
\hline Umbrella sampling & 0 & 9.0 & 7.7 & 9.0 & 2.7 \\
\hline
\end{tabular}


Table 2. Structural comparison of significant points along the free energy pathways from the intercalated state (IC), through the intermediate (IM) state, to the minor groove-bound state (MG1). All parameters were calculated using Curves+.. ${ }^{51}$ The minor groove width is measured midway between the C6 and G7 base pairs.

(A) Metadynamics

\begin{tabular}{|l|l|l|l|l|l|}
\hline Parameters & IC & TS1 & IM & TS2 & MG1 \\
\hline Rise C6pG7 $(\AA)$ & 7.4 & 7.3 & 6.0 & 5.5 & 3.8 \\
\hline Roll C6pG7 $\left(^{\circ}\right)$ & -2 & 28 & 44 & 42 & 8 \\
\hline$\sum$ Roll A5-T8 $\left(^{\circ}\right)$ & -2 & 40 & 45 & 44 & 7 \\
\hline Twist C6pG7 $\left(^{\circ}\right)$ & 38 & 25 & 28 & 29 & 39 \\
\hline$\sum$ Twist A5-T8 $\left(^{\circ}\right)$ & 81 & 74 & 84 & 84 & 99 \\
\hline Minor groove width $(\AA)$ & 9.5 & 10.4 & 10.9 & 10.5 & 6.9 \\
\hline A-ring pucker & Eq & Eq & Eq & Ax & Ax \\
\hline
\end{tabular}

(B) Umbrella sampling

\begin{tabular}{|l|l|l|l|l|l|}
\hline Parameters & IC & TS1 & IM & TS2 & MG1 \\
\hline Rise $\left(^{\circ}\right)$ & 7.4 & 7.2 & 4.2 & 3.6 & 3.8 \\
\hline Roll C6pG7 $\left(^{\circ}\right)$ & 0 & 6 & 39 & 2 & -4 \\
\hline$\sum$ Roll A5-T8 $\left(^{\circ}\right)$ & -9 & 8 & 32 & 6 & -4 \\
\hline Twist C6pG7 $\left(^{\circ}\right)$ & 34 & 24 & 14 & 35 & 41 \\
\hline$\sum$ Twist A5-T8 $\left(^{\circ}\right)$ & 87 & 77 & 74 & 95 & 99 \\
\hline Minor groove width $(\AA)$ & 7.0 & 9.0 & 11.3 & 6.0 & 4.9 \\
\hline A-ring pucker & Eq & Strained & Ax & Ax & Eq \\
\hline
\end{tabular}




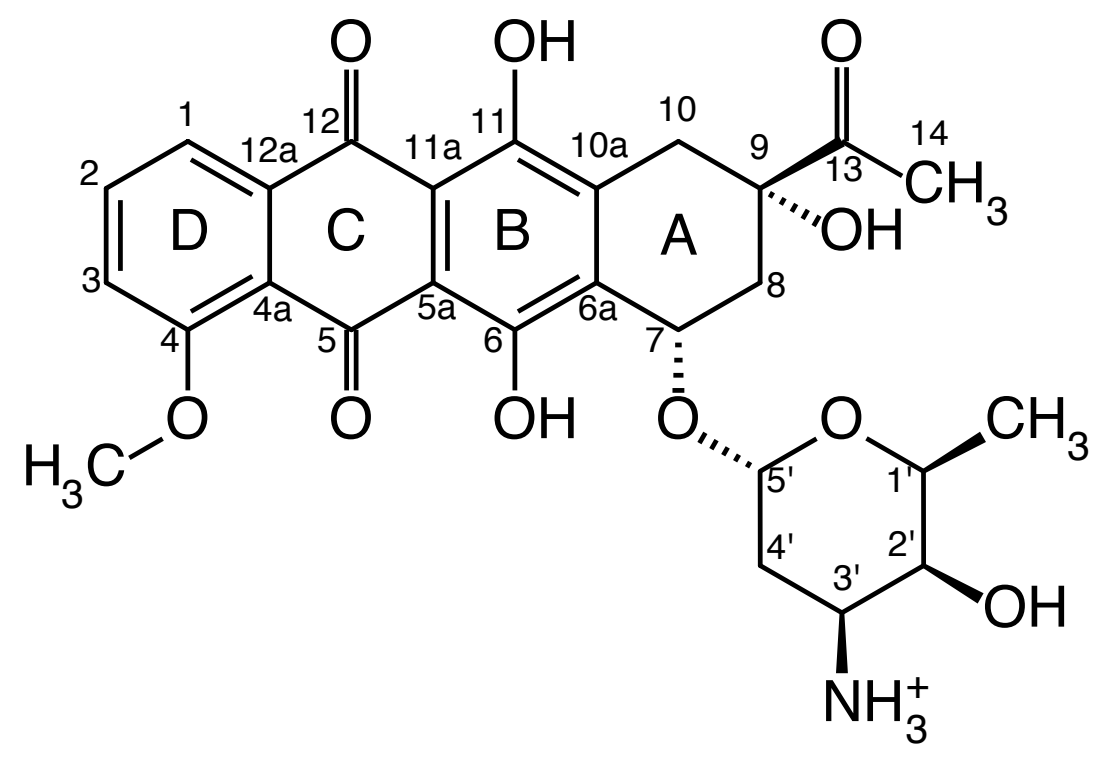

Figure 1. Chemical structure and notation of daunomycin. The rings B, C and D constitute the planar, conjugated anthraquinone moiety that intercalates between adjacent DNA base pairs. The A-Ring is non-planar and flexible and carries the cationic daunosamine sugar at position $\mathrm{C} 7$. 


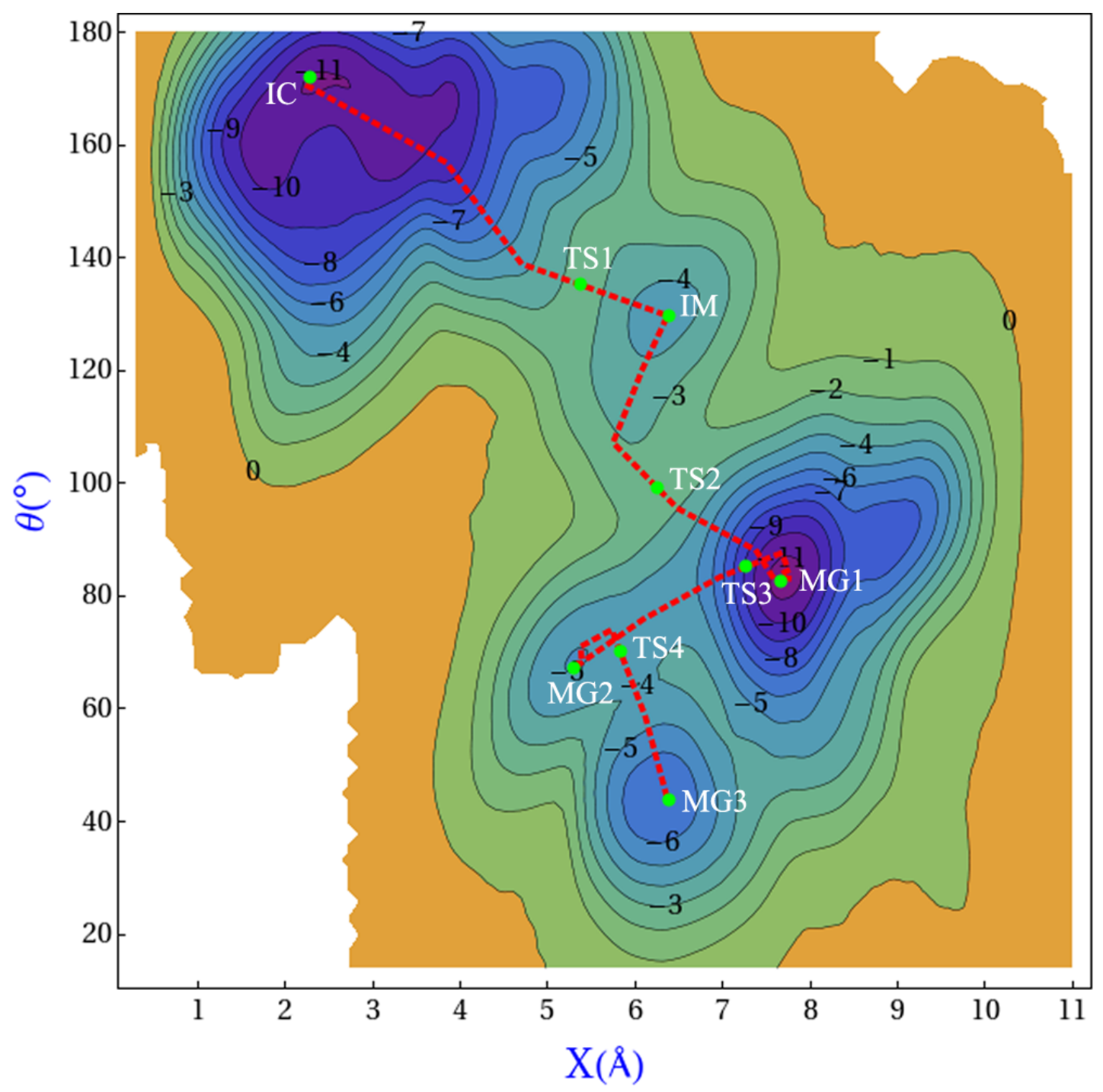

Figure 2. 2D contour plot of the metadynamics free energy trajectory obtained using three collective variables $(\mathrm{X}, \theta$, and $\mathrm{Y})$. Values corresponding to displacements along the Y-axis (roughly aligned with the DNA helical axis) have been Boltzmannaveraged. Free energy contours are drawn at $1 \mathrm{kcal} \mathrm{mol}^{-1}$ intervals and colored from yellow to purple as the system becomes more stable. The lowest free energy path through the most important local minima and the corresponding transition states is shown. 

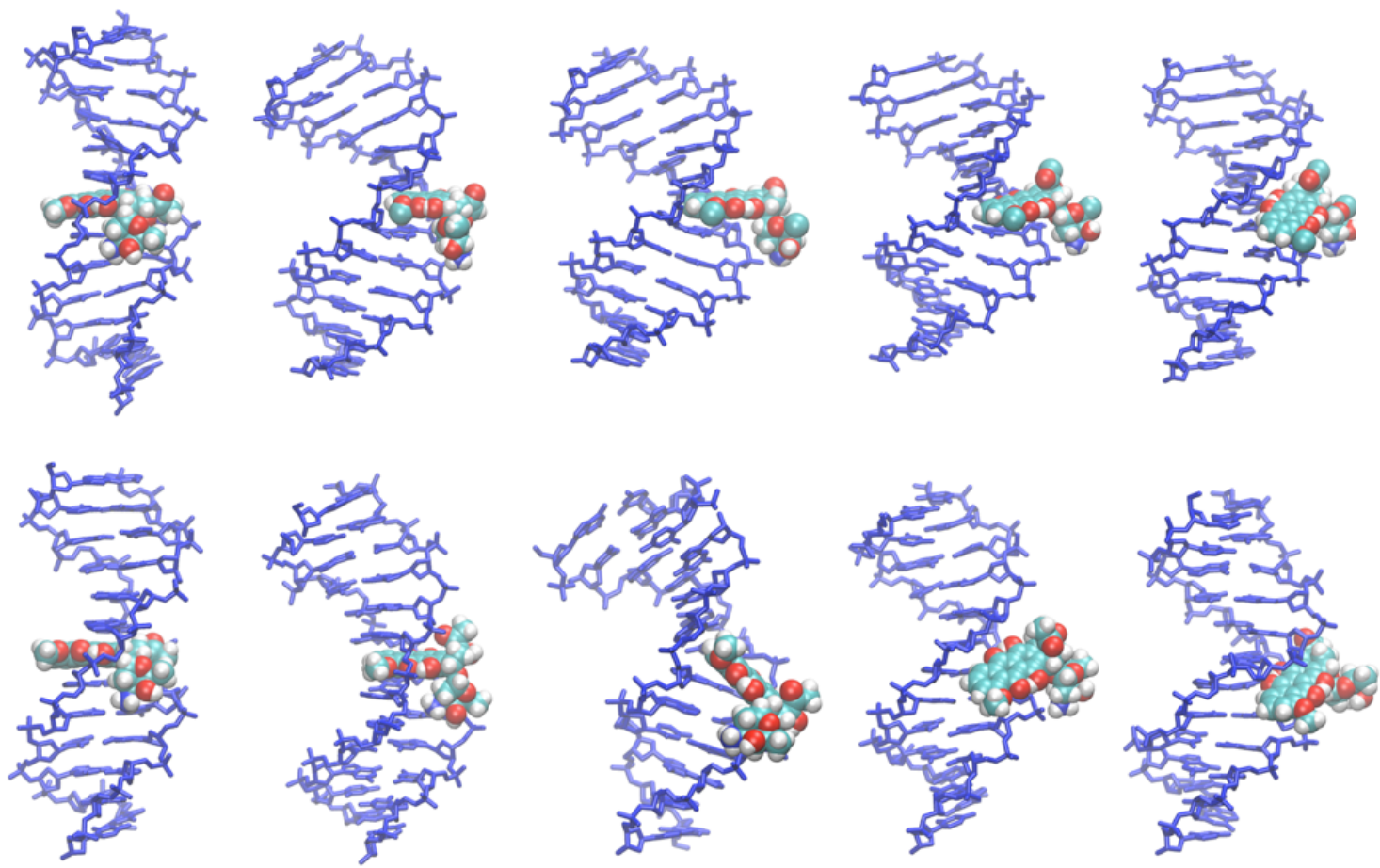

Figure 3. Representative structures from the intercalation site to the minor groovebound site (left to right: IC, TS1, IM, TS2, MG1) for the metadynamics (above) and umbrella-sampling (below) pathways. DNA is shown as a blue Dreiding model and daunomycin as a CPK model with standard chemical coloring. 
(A)

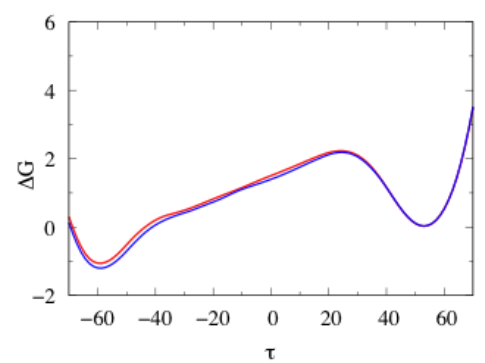

(B)

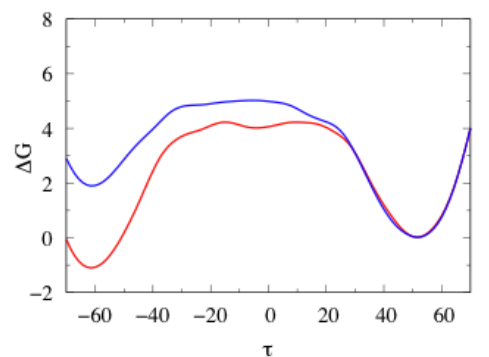

(C)

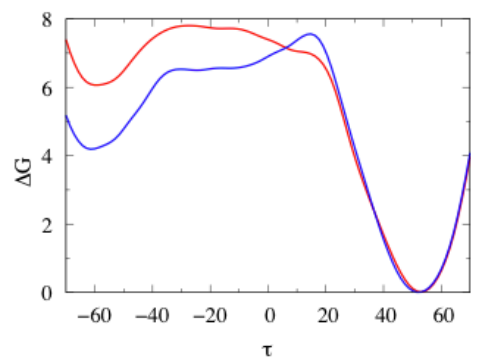

Figure 4. Free energy profiles ( $\mathrm{kcal} \mathrm{mol}^{-1}$ ) for repuckering the A-ring of daunomycin using the dihedral $\tau=\mathrm{C} 7-\mathrm{C} 8-\mathrm{C} 9-\mathrm{C} 10$ (degrees). Local free energy minima are found at $\tau \approx 50^{\circ}$ (corresponding to an equatorial position of the C13 acetyl group) and at $\tau \approx$ $-60^{\circ}$ (corresponding to an axial position of the acetyl group): (A) daunomycin in aqueous solution; (B) daunomycin bound to the minor groove of DNA; (C) daunomycin intercalated within at the C6pG7 step of DNA. In each case, the red curve was obtained in minimal salt conditions and the blue curve in "physiological" conditions $(0.15 \mathrm{M} \mathrm{NaCl})$. 
(A)

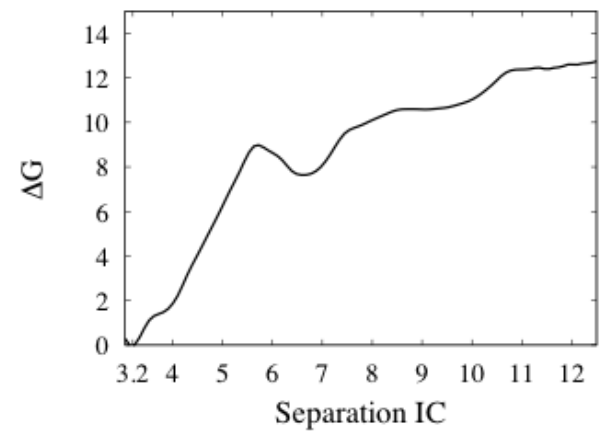

(C)

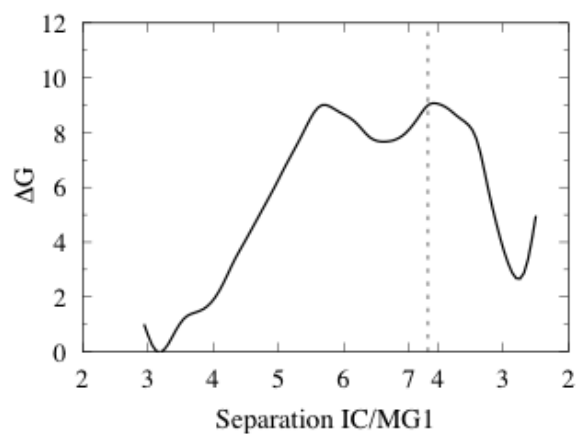

(B)

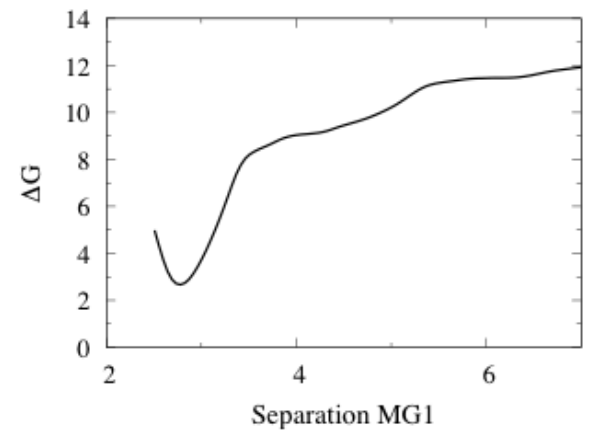

(D)

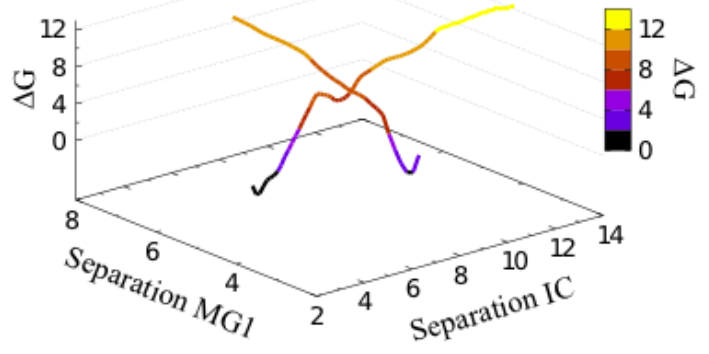

Figure 5. Umbrella-sampling free energy pathways (energies in $\mathrm{kcal}^{\mathrm{mol}} \mathrm{l}^{-1}$ and separation distances in $\AA$ ): (A) separation from the intercalated state (IC), showing the intermediate state (IM); (B) separation from the minor groove-bound state (MG1); (C) assembly of a single pathway between IC and MG1 at the point indicated by the vertical dotted line; (D) 3D view of the intersection of the two free energy pathways. 
(A)

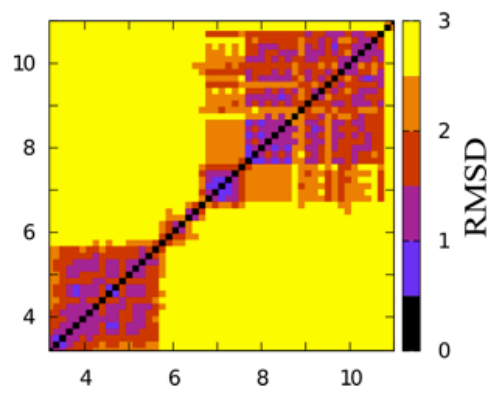

(B)

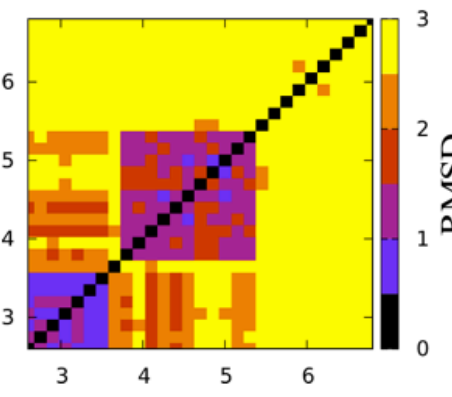

(C)

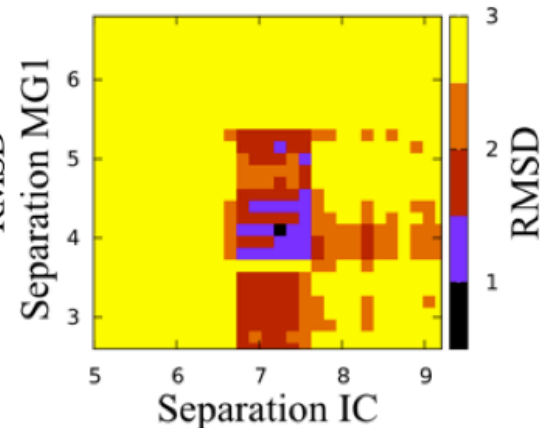

Figure 6. 2D RMSD plots $(\AA)$ between DNA-daunomycin conformations drawn from the umbrella-sampling pathways. The numbers along the axes show the increasing separation restraints and smaller RMSD differences are shown by darker colors. Dark patches centered on the diagonal indicate similar structures which persist for a range of separation distances: (A) conformations along the pathway from the intercalated state (IC); (B) conformations along the pathway from the minor groovebound state (MG1); (C) between conformations from the two pathways, showing the common structures sampled at $7.25 \AA$ along the IC pathway and at $4.1 \AA$ along the MG1 pathway. 
(A)

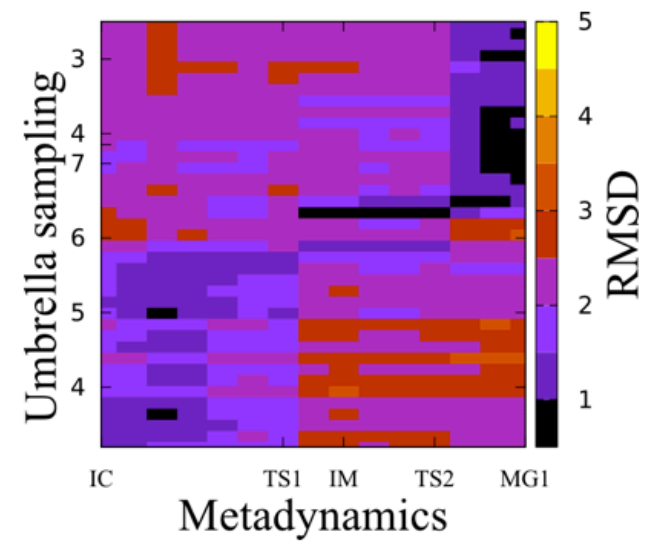

(B)

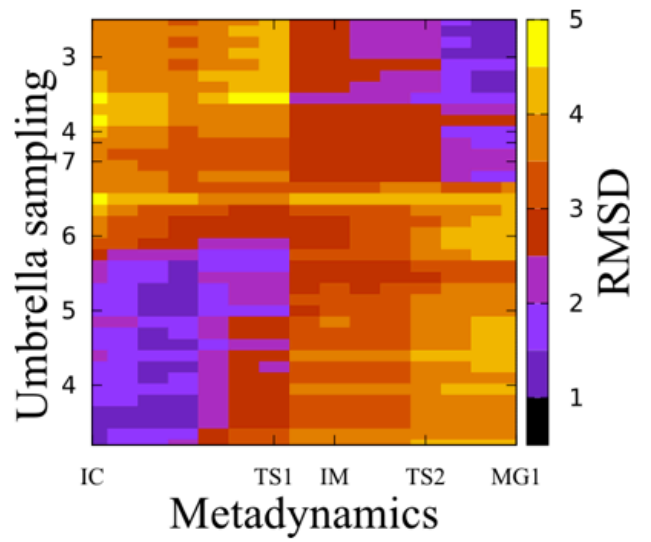

Figure 7. 2D RMSD plots $(\AA)$ between conformations drawn from the metadynamics pathway (horizontal axis) and the umbrella-sampling pathway (vertical axis): (A) for DNA alone; (B) for the DNA-daunomycin complex. See the caption to Figure 6 for further explanations. 


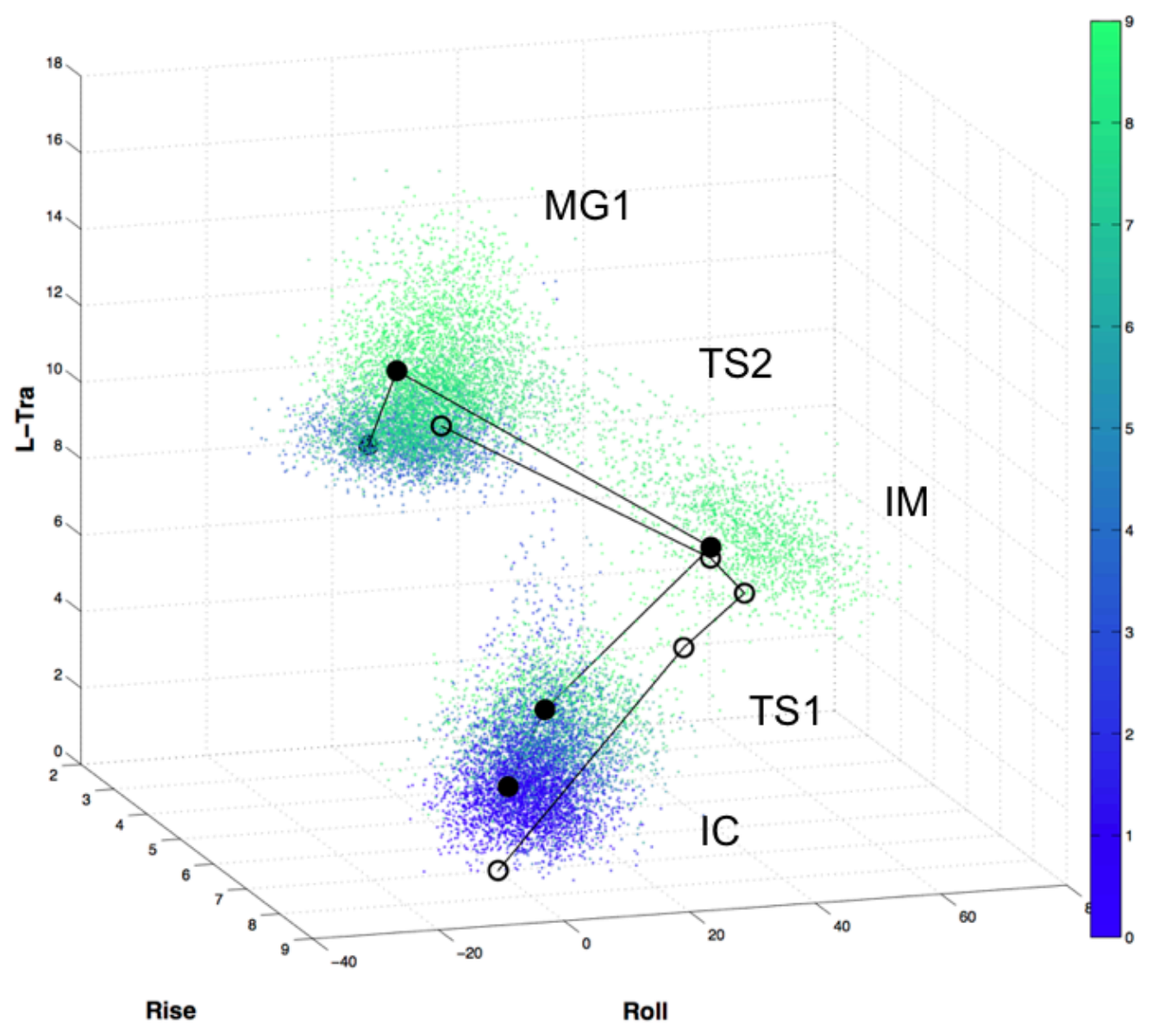

Figure 8. Pathway sampling from the minor groove site to the intercalation site. The 3D plot shows the rise and roll of DNA at the C6pG7 intercalation step (horizontal plane) and the drug to center of DNA axis distance, L-Tra (vertical axis). Snapshots from the umbrella-sampling pathway are shown as points colored from blue to green (increasing free energy with respect to the intercalated state, see color bar in kcal mol '). The main states along the pathway are labeled: IC (high rise and low roll), IM (high positive roll), MG1 (low rise and high L-Tra), TS1 (separating IC and IM) and TS2 (separating IM and MG1) are the less densely sampled arms joining the main clusters. Representative structures from the metadynamics and umbrella-sampling pathways are shown as open and filled circles respectively. 